\title{
Magnetic molecularly imprinted polymer for the isolation and detection of biotin and biotinylated biomolecules
}

\author{
A. Ben Aissa ${ }^{\mathrm{a}, 1}$, A. Herrera-Chacon ${ }^{\mathrm{a}, 1}$, R.R. Pupin ${ }^{\mathrm{b}}$, M.D.P.T. Sotomayor ${ }^{\mathrm{b}}$, M.I. Pividori ${ }^{\mathrm{a}, *}$ \\ a Grup de Sensors i Biosensors, Departament de Química, Universitat Autònoma de Barcelona, Bellaterra, Spain \\ ${ }^{\mathrm{b}}$ UNESP - Univ Estadual Paulista, Institute of Chemistry, Department of Analytical Chemistry, Araraquara, SP, Brazil
}

\section{A R T I C L E I N F O}

\section{Article history:}

Received 16 June 2016

Received in revised form

26 July 2016

Accepted 27 July 2016

Available online 28 July 2016

Keywords:

magnetic particles

magnetic molecularly-imprinted polymer

biotinylated biomolecules

magneto-actuated immunoassay

immunomagnetic separation

\begin{abstract}
A B S T R A C T
Magnetic separation based on biologically-modified magnetic particles is a preconcentration procedure commonly integrated in magneto actuated platforms for the detection of a huge range of targets. However, the main drawback of this material is the low stability and high cost. In this work, a novel hybrid molecularly-imprinted polymer with magnetic properties is presented with affinity towards biotin and biotinylated biomolecules. During the synthesis of the magneto core-shell particles, biotin was used as a template. The characterization of this material by microscopy techniques including SEM, TEM and confocal microscopy is presented. The application of the magnetic-MIPs for the detection of biotin and biotinylated DNA in magneto-actuated platforms is also described for the first time. The magneticMIP showed a significant immobilization capacity of biotinylated molecules, giving rise to a cheaper and a robust method (it is not required to be stored at $4{ }^{\circ} \mathrm{C}$ ) with high binding capacity for the separation and purification under magnetic actuation of a wide range of biotinylated molecules, and their downstream application including determination of their specific targets.
\end{abstract}

(c) 2016 Elsevier B.V. All rights reserved.

\section{Introduction}

Since the early reports on magnetic separation technology (Rembaum et al., 1982), magnetic particles (MPs) have been used as a powerful and versatile preconcentration tool in a variety of analytical and biotechnology applications (Reddy et al., 2012). This technology has been widely incorporated for researchers in classical methods including PCR and immunoassays, and in emerging technologies such as biosensors and microfluidic devices with application ranging from biomarker detection of infectious diseases (Carinelli et al., 2015) foodborne pathogens (Brandão et al., 2015a), among others. Magnetic particles have been commercially available for many years. They consist of an inorganic core of magnetic materials coated with a polymer which confers stability (such as polystyrene, dextran, polyacrylic acid, or silica), providing also addressable functional groups to favour the immobilization of biomolecules. Hence, magnetic particles can be coupled to peptides, proteins, antibodies and nucleic acids. Among the different biological biomolecules, strept(avidin)-modified MPs are widely used due to the specific interaction with biotin and biotinylated biomolecules. Avidin and streptavidin bind four moles of biotin per

\footnotetext{
* Corresponding author.

E-mail address: isabel.pividori@uab.cat (M.I. Pividori).

${ }^{1}$ These authors contributed equally to this work.
}

mole of protein with an extraordinary affinity $\left(\mathrm{Ka}=10^{15} \mathrm{M}^{-1}\right)$ (Savage et al., 1992). Some applications in which the strept(avidin)-biotin interaction have been used include ELISA, immunohistochemical staining, Western, Northern and Southern blotting, immunoprecipitation, cell-surface labelling, affinity purification, fluorescence-activated cell sorting (FACS), among many others (Meier and Fahrenholz, 1996). The valeric acid side chain of the biotin molecule can be derivatised to incorporate reactive groups that are used to attach biotin to biomolecules, including peptides, antibodies, enzymes, receptors, nucleic acids, without significantly altering their biological activity (Savage et al., 1992). To take advantages of this interaction for separation and purification of biotinylated biomolecules, as well as for downstream applications, there are many commercial available avidin/streptavidin magnetic particles, for instance, Streptavidin MagBeads (GenScript), Dynabeads ${ }^{\mathrm{TM}} \mathrm{M}-280$ Streptavidin (Dynal), hyBeads ${ }^{\circledR}$ Streptavidin (Hyglos), MasterBeads Streptavidin (Ademtech), among others. The use of MPs greatly improves the performance of the biological reaction by increasing the surface area, enhancing the washing steps and, importantly, minimizing the matrix effect. In addition, MPs can be easily magneto-actuated using permanent magnets (Pividori and Alegret, 2005). Beside the amazing properties and the huge range of applications, the main drawback of the biologically-modified MPs is their high cost and low stability at harsh conditions. Molecularly Imprinted Polymers (MIPs) are synthetic biomimetic materials mimicking biological receptors 
(Vlatakis et al., 1993; Ramstrom and Mosbach, 1999). They are highly cross-linked macromolecular structures towards the template which is then extracted after polymerization, originating cavities (binding sites) complementary to the template molecule (Moreno-Bondi et al., 2008), acting as plastic antibodies (Haupt, 2010). Although MIPs have in general lower affinity and selectivity compared to the biological counterparts, they show important technological features: i) they can be easily and affordably synthesized on a animal-free large scale procedures, and ii) they show high chemical and mechanical stability, allowing to work in harsh conditions ( $\mathrm{pH}$, temperature, solvents). Due to their wide range of applications, the synthesis of streptavidin-mimicking molecularly imprinted polymers has been previously reported by using biotin as a template and different monomers (Takeuchi et al., 2000; Piletska et al., 2004; Wiklander et al., 2011). This paper described the synthesis of magneto-actuated molecularly imprinted polymers (magnetic-MIPs) as well as the separation of biotinylated biomolecules, and the downstream applications including detection of their targets with fluorescence and optical readouts. The preparation of the magnetic-MIP is based on a core-shell synthesis in which the core was made of magnetite recovered by a shell of the MIP (Chen, 2009), using biotin as a template. The characterization of the material was performed by SEM, TEM, Energy-dispersive $\mathrm{X}$-ray spectroscopy and confocal microscopy. The binding capacity towards biotin and biotinylated biomolecules including enzymes, dendrimers and DNA is demonstrated for the first time and compared with the commercially-available streptavidin MPs. Moreover, the integration of this material in magneto-immunoassay is also demonstrated for the first time. The magneticMIP is a cheaper and robust alternative to the biologically-modified magnetic particles (streptavidin-MPs), showing the synergic advantages of MIPs and MPs, such as low cost of production, stability and magnetic actuation, and presenting promising features for their integration in magneto-actuated approaches, including biosensors and microfluidic devices for the detection of a wide variety of targets.

\section{Experimental section}

\subsection{Instrumentation}

The SEM images were taken with the scanning electron microscope EVO MA-10 (with EDS Detector, Oxford LINCA). The TEM images were taken with the transmission electron microscope JEM-2011 (with EDS Detector Oxford LINCA). The confocal fluorescence images were taken with the TCP-SP5 Leica Microscope. Optical measurements were performed on a TECAN Sunrise microplate reader with Magellan v4.0 software. The PCR reaction was carried out in a thermal cycler (Applied Biosystems, Life Technologies Corporation). Further description is provided in Supp. data.

\subsection{Chemicals and Biochemicals}

The reagents for the synthesis of the magnetic particles based on biotin molecularly imprinted polymer (magnetic-MIP) and the corresponding NIP were purchased from Sigma Aldrich ${ }^{\circledR}$, while 2,2'azobisisobutyronitrile (AIBN), from Fisher ${ }^{\circledR}$. ELISA Substrate kit (Prod. $\mathrm{N}^{\circ}$ 34021) was purchased from Thermo Scientific. The streptavidin labeled with cyanine 5 dye (Strep-Cy5) was purchased from Life Technologies (Prod No. SA-1011), while the fluorescent dye Atto 665-biotin, from Sigma Aldrich (Prod № 1376). The reagents for the magneto actuated immunoassay were biotin-HRP (Fisher Scientific, Prod. $\mathrm{N}^{\circ}$ 10324693) and antiDIG-HRP (anti-digoxigenin-POD Fab fragments, Roche Diagnostics, Prod. $\mathrm{N}^{\circ}$ 11207733910 ). All buffer solutions were prepared with milliQ water and all other reagents were in analytical reagent grade (supplied from Sigma and Merck). The composition of these solutions is described in Supp. data, Table S1. The primers for the double-tagging PCR of E. coli were obtained from TIB-Molbiol $\mathrm{GmbH}$ (Berlin, Germany), and the sequences are detailed in Supp. data, Table S2.

\subsection{Synthesis of the magnetic molecularly imprinted polymer (magnetic-MIP) for biotin}

The preparation of the magnetic-MIP (schematized in Fig. S1, Supp. data) was based on a core-shell synthesis in which the core was made of magnetite recovered by a shell of the MIP using biotin as a template (Pividori and Sotomayor, 2016). The core-shell synthesis (UzuriagaSánchez et al., 2016), with slight modifications is described in detail in Supp. data (and Figs. S2 to S4 therein). The magnetic-NIP was used in all cases as a control of the non-specific adsorption of the biotinylated biomolecules. The suspension of the magnetic-MIP in TRIS buffer $\mathrm{pH}$ 7.4 can be easily separated with the help of a magnet (as shown in Supp. data, video 1 and 2, and Fig. S5).

\subsection{SEM and TEM study of the magnetic-MIP}

The characterization of the products of the synthesis was performed by scanning electron microscopy (SEM) operated at $3 \mathrm{kV}$ and transmission electron microscopy, (TEM) operated at $200 \mathrm{kV}$. After each step of the core-shell synthesis, the product was collected and characterized by SEM and TEM, as detailed in Supp. data. Energy dispersive X-ray spectroscopy detector (EDS) was also used for the elemental analysis.

\subsection{Characterization of the binding of biotinylated biomolecules by confocal microscopy}

The evaluation of the binding of biotinylated biomolecules tagged with fluorophores (including atto 665-biotin and a G4 biotin-dendrimer conjugated with streptavidin-Cy5) were performed by confocal microscopy, as schematically shown in Fig. 1, panels $A$ and $B$, respectively. In the first experiment, the magneticMIP was reacted with atto 665-biotin. To achieved this $0.2 \mathrm{mg}$ of magnetic-MIP and $1 \mu \mathrm{L}$ of atto 665 -biotin $\left(1 \mathrm{mg} \mathrm{mL}^{-1}\right)$ were incubated in $200 \mu \mathrm{L}$ TRIS buffer $\mathrm{pH} 7.4$ for 30 min with shaking at $37{ }^{\circ} \mathrm{C}$. A washing step was then performed for $5 \mathrm{~min}$ in TRIS buffer $\mathrm{pH}$ 7.4. The negative control was performed with the magneticMIP without the addition of the conjugate. Another experiment was performed by the incubation of the magnetic-MIP with a G4 biotinylated dendrimer (96 biotin molecules per molecule of dendrimer) followed by the incubation with streptavidin-Cy5. In this approach, $10 \mu \mathrm{L}$ of biotinylated dendrimer $\left(1 \mathrm{mg} \mathrm{mL}^{-1}\right.$ in $2 \%$ DMSO) and $2 \mathrm{mg}$ of magnetic-MIPs were incubated in $200 \mu \mathrm{L}$ TRIS buffer pH 7.4 for 30 min with shaking at $37^{\circ} \mathrm{C}$. The magnetic-MIP was separated from the supernatant with a magnet. After that, $200 \mu \mathrm{L}$ of streptavidin-Cy5 $\left(2 \mu \mathrm{g} \mathrm{mL}^{-1}\right)$ was added and the mixture was incubated again for $30 \mathrm{~min}$ at $37^{\circ} \mathrm{C}$, followed by a washing step. The negative control was similarly performed but avoiding the addition of the biotinylated dendrimer. In both cases, the images were scanned in two dimensions along $\mathrm{x}$ and $\mathrm{y}$ axes by using the laser AOTF at a wavelength of $633 \mathrm{~nm}$ whereas the emission was captured in the range of $645-785 \mathrm{~nm}$.

\subsection{Characterization of the binding of biotin-HRP by a magneto- actuated immunoassay}

The binding of biotinylated biomolecules tagged with HRP as optical reported (including biotin-HRP and double-tagged DNA conjugated with an antibody antiDIG-HRP) were evaluated by a 


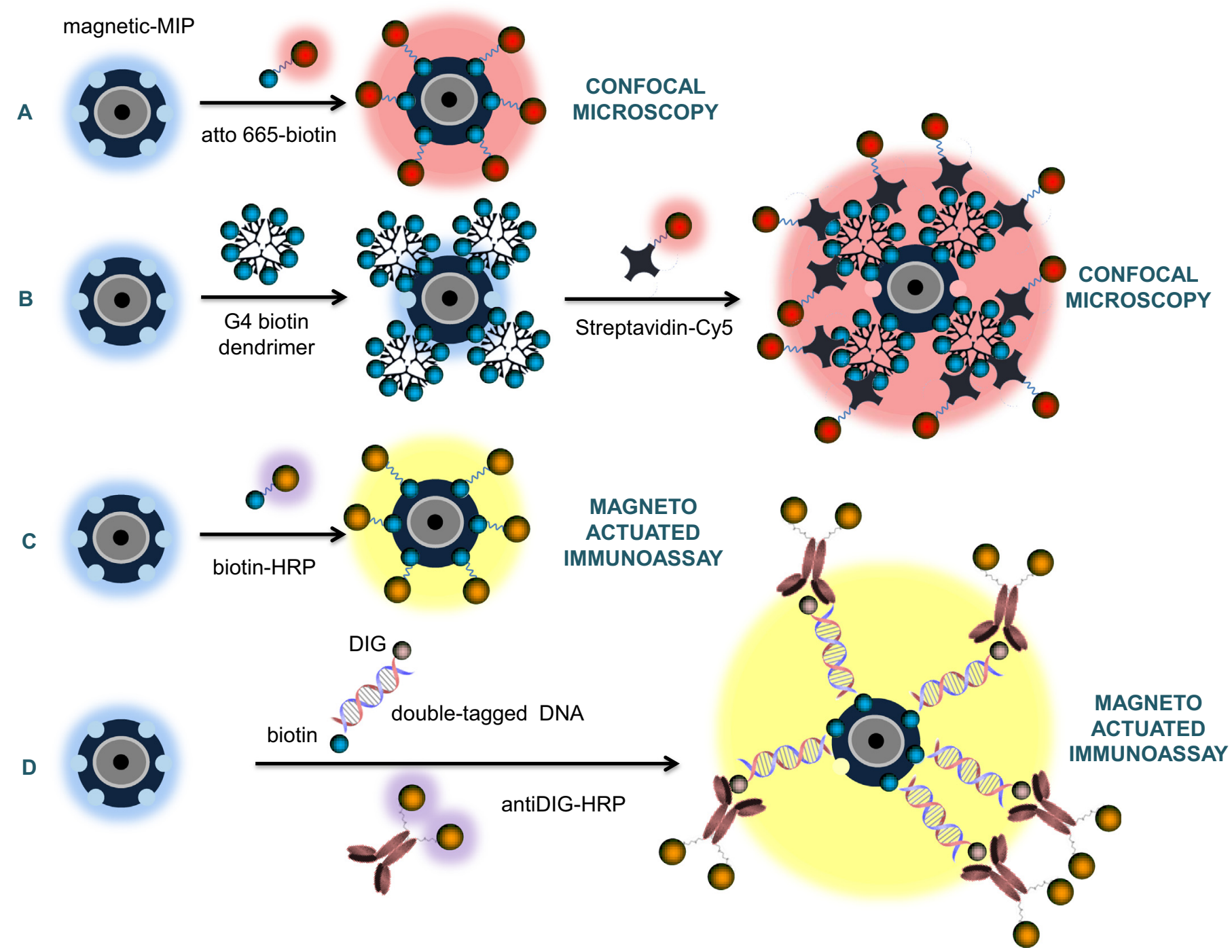

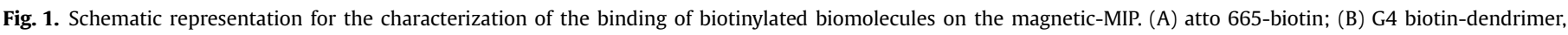

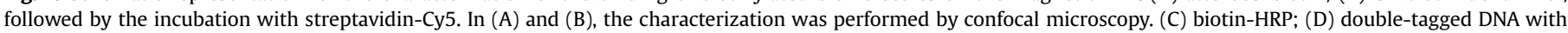

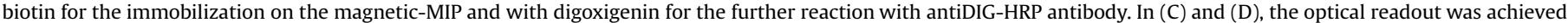
upon the addition of the substrate for the HRP in a magneto-actuated immunoassay.

magneto-actuated immunoassay, as schematically shown in Fig. 1, panels $C$ and $D$, respectively.

The magneto-actuated immunoassay for biotin-HRP (Fig. S6, Supp. data) was performed in 96-well microtiter plates and involved the following steps: (A) Incubation with biotin-HRP conjugate. In this step, the magnetic-MIP ( $0.55 \mathrm{mg}$ per well) was incubated with $100 \mu \mathrm{L}$ of biotin-HRP (ranging from 0 to $100 \mathrm{ng} \mathrm{mL}^{-1}$ ) in TRIS buffer $\mathrm{pH} 7.4$ for $30 \mathrm{~min}$ with shaking at room temperature. (B) Washing step with $100 \mu \mathrm{L}$ of TRIS buffer $\mathrm{pH}$ 7.4 for $5 \mathrm{~min}$ (C) Optical readout with $100 \mu \mathrm{L}$ of substrate solution $\left(0.004 \% \mathrm{v} / \mathrm{v} \mathrm{H}_{2} \mathrm{O}_{2}\right.$ and $0.01 \% \mathrm{w} / \mathrm{v}$ TMB in citrate buffer) incubated for $30 \mathrm{~min}$ at RT under dark conditions. The enzymatic reaction was stopped by adding $100 \mu \mathrm{L}$ of $\mathrm{H}_{2} \mathrm{SO}_{4}\left(2 \mathrm{~mol} \mathrm{~L}^{-1}\right)$. The absorbance measurement of the supernatants was thus performed with the microplate reader using a $450 \mathrm{~nm}$ filter. After each incubation or washing step, a 96-well magnet plate separator was positioned under the microtiter plate until pellet formation on the bottom corner, followed by supernatant separation, as shown in Supp. data, video 3 . The incubation time was studied. The assay was performed in a similar manner as above, but by using $0.33 \mathrm{mg}$ per well of the magnetic-MIP upon two different conditions for incubation: 30 and $120 \mathrm{~min}$, in a concentration for biotin-HRP ranging from 0 to $125 \mathrm{ng} \mathrm{mL}^{-1}$. Finally, as the $\mathrm{pH}$ and the buffer composition is an important parameter for the binding, beside TRIS buffer $\mathrm{pH} 7.4$, different buffer composition and $\mathrm{pH}$, summarized in Table S1, were tested, including citrate $\mathrm{pH} 6.4$ and 7.4, phosphate $\mathrm{pH} 6.4$ and 7.4, borate $\mathrm{pH} 7.4$ and 8.4, and TRIS pH 7.4 and 8.4. The assay was performed by using the different buffers in a similar manner as above, but by using $0.33 \mathrm{mg}$ per well of the magnetic-MIP. The magnetic-NIP was used as a control of the nonspecific adsorption of the biotinylated biomolecules.

\subsection{Characterization of the binding of double-tagged DNA from $E$. coli 0157:H7 by a magneto-actuated immunoassay}

The double-tagged DNA from E. coli 0157: H7 was achieved by PCR performed with a double-tagging set of primers for the amplification of the eaeA (151 bp) gene fragment specific to $E$. coli (Brandão et al., 2015b), being each primer labeled in 5' with biotin (to achieve the immobilization on the magnetic-MIP) and digoxigenin (for the optical readout by using the optical reporter antiDIG-HRP), as schematically shown in Fig. 1, panel D. The detailed conditions for the double-tagging PCR is presented in Supp. data and Figures therein, including the bacterial strain, growth 
condition and DNA extraction, safety considerations, the sequence of the tagging-primers (Table S2), and the procedure for the double-tagging PCR (Table S3 and Fig. S7). The detection of the double-tagged DNA amplicon was performed in a magneto-actuated immunoassay (Fig. S8, Supp. data) in 96-well microtiter plates, involving the following steps: (A) One-step incubation with double-tagged amplicon and AntiDIG-HRP conjugate. In this step, the magnetic-MIP ( $0.55 \mathrm{mg}$ per well $)$ was incubated with $50 \mu \mathrm{L}$ of amplicon (ranging from 0 to $206 \mathrm{ng} \mathrm{mL}^{-1}$ ) and $1.35 \mu \mathrm{g}$ of antiDIGHRP in TRIS $0.05 \%$ Tween buffer solution $\mathrm{pH} 7.4$ for 30 min with shaking at room temperature. (B) A washing step with $100 \mu \mathrm{L}$ of TRIS buffer $\mathrm{pH}$ 7.4. (C) Optical readout as previously described for biotin-HRP. The magnetic-NIP was also used as a control of the non-specific adsorption.

\subsection{Quantification of biotin based on a competitive magneto-ac- tuated immunoassay}

The quantification of biotin was performed by a competitive magneto-actuated immunoassay in two steps, as shown in Fig. S23 (Supp. data), in which biotin competes with biotin-HRP for the binding sites of the magnetic-MIP. The competitive magneto-actuated immunoassay for biotin was performed in 96-well microtiter plates and involved the following steps: (A) Incubation with biotin-HRP conjugate. In this step, the magnetic-MIP $(0.55 \mathrm{mg}$ per well) was incubated with $100 \mu \mathrm{L}$ of biotin-HRP $\left(100 \mathrm{ng} \mathrm{mL}^{-1}\right)$ in TRIS buffer $\mathrm{pH} 7.4$ for 30 min with shaking at room temperature. (B) Washing step with $100 \mu \mathrm{L}$ of TRIS buffer $\mathrm{pH} 7.4$, for $5 \mathrm{~min}$ (C) Competitive reaction with $100 \mu \mathrm{L}$ of biotin, ranging from $0.2 \mathrm{pg} \mathrm{mL}^{-1}$ to $1.9 \mathrm{ng} \mathrm{mL}^{-1}$ in TRIS buffer $\mathrm{pH} 7.4$ for $30 \mathrm{~min}$ with shaking at room temperature, followed by a washing step as above. (D) Optical readout as previously described for biotin-HRP.

Different competition formats were tested as detailed in Supp. data, including a i) one-simultaneous competition step (30 min) (Fig. S17); ii) one consecutive-competition step by the incubation of biotin (30,120 and $240 \mathrm{~min}$ ) and biotin-HRP (30 min) (Fig. S19); iii) one consecutive-competition step by the incubation of biotinHRP (30 min) and biotin (30 and $120 \mathrm{~min}$ ) (Fig. S21).

\section{Results and discussion}

\subsection{SEM and TEM study of the magnetic-MIP}

The characterization of the products of the synthesis was performed by scanning electron microscopy (SEM) and transmission electron microscopy (TEM). After each step of the core-shell synthesis, the product was collected and submitted to SEM, TEM and elemental analysis. The results for each synthetic step are presented in Supp. data, Fig. $\mathrm{S} 9$ for $\mathrm{Fe}_{3} \mathrm{O}_{4}$, Fig. $\mathrm{S} 10$ for $\mathrm{Fe}_{3} \mathrm{O}_{4} @ \mathrm{SiO}_{2}$ and Fig. $\mathrm{S} 11$ for $\mathrm{Fe}_{3} \mathrm{O}_{4} @ \mathrm{SiO}_{2}$-MPS. Finally, Fig. S12 shows the results for the magneticMIP and NIP. The SEM and TEM images revealed irregular spherical shape nanoparticles (average size $371 \mathrm{~nm} / \mathrm{SD} 101 \mathrm{~nm}$ ) highly crosslinked among them. Fig. 2 shows comparatively the aspect of the magnetic-MIP (panel A) and the magnetic-NIP (panel B). As can be observed, during the polymerization of the MIP, the polymer includes many nanoparticles of magnetite in the structure, in agreement with the high magnetization of the material shown in videos 1 and 2 (Supp. data). It is important to highlight from the TEM images the different porosity pattern of the magnetic-MIP compared with the corresponding NIP. The porosity can be attributed to the cavities towards the biotin template which were achieved during the template extraction. Other characterization of the material was previously performed, including FTIR, magnetic hysteresis, XRD, SEM and $\mathrm{N}_{2}$-sorption measurements (Uzuriaga-Sánchez et al., 2016).

\subsection{Characterization of the binding of biotinylated biomolecules by confocal microscopy}

The binding of biotinylated biomolecules tagged with fluorophores (including atto 665-biotin and a G4 biotin-dendrimer conjugated with streptavidin-Cy5) were studied by confocal microscopy, as schematically shown in Fig. 1, panels A and B, respectively. In the first experiment (Fig. 3, Panels B and C, as well as the negative control, panel A), the magnetic-MIP was attached with atto 665-biotin (MW $1046 \mathrm{~g} \mathrm{~mol}^{-1}$ ), a small molecule that can freely enters in the cavities of the magnetic-MIPs. This experiment suggests a high binding capacity of the magnetic-MIP towards the biotinylated dye, since the material is completely embedded of the dye. The other experiment (Fig. 3, Panels E and F, as well as the negative control, panel D) was
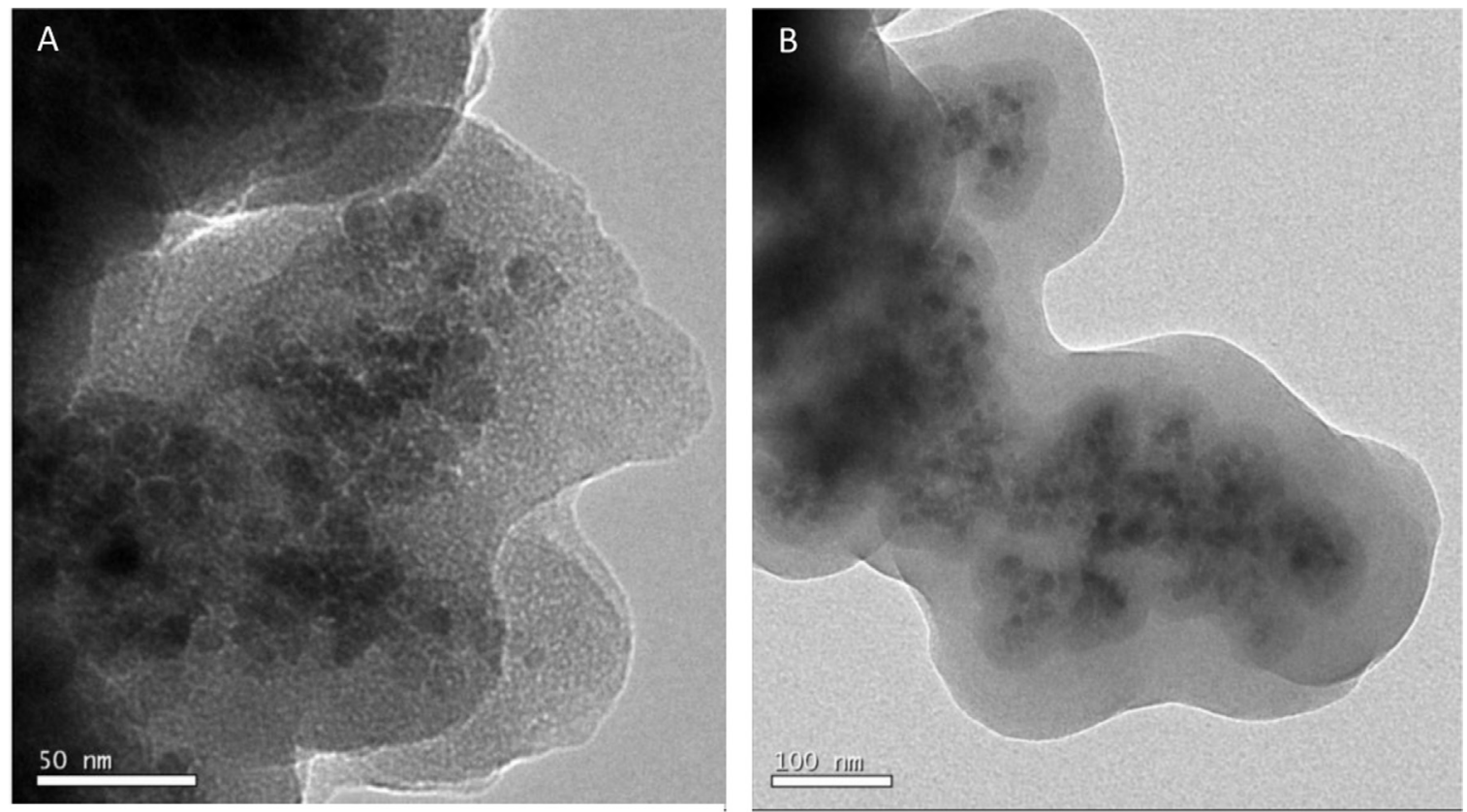

Fig. 2. Comparative study at high resolution of magnetic-MIP (A) and NIP (B) by TEM operated at $200 \mathrm{kV}$. 

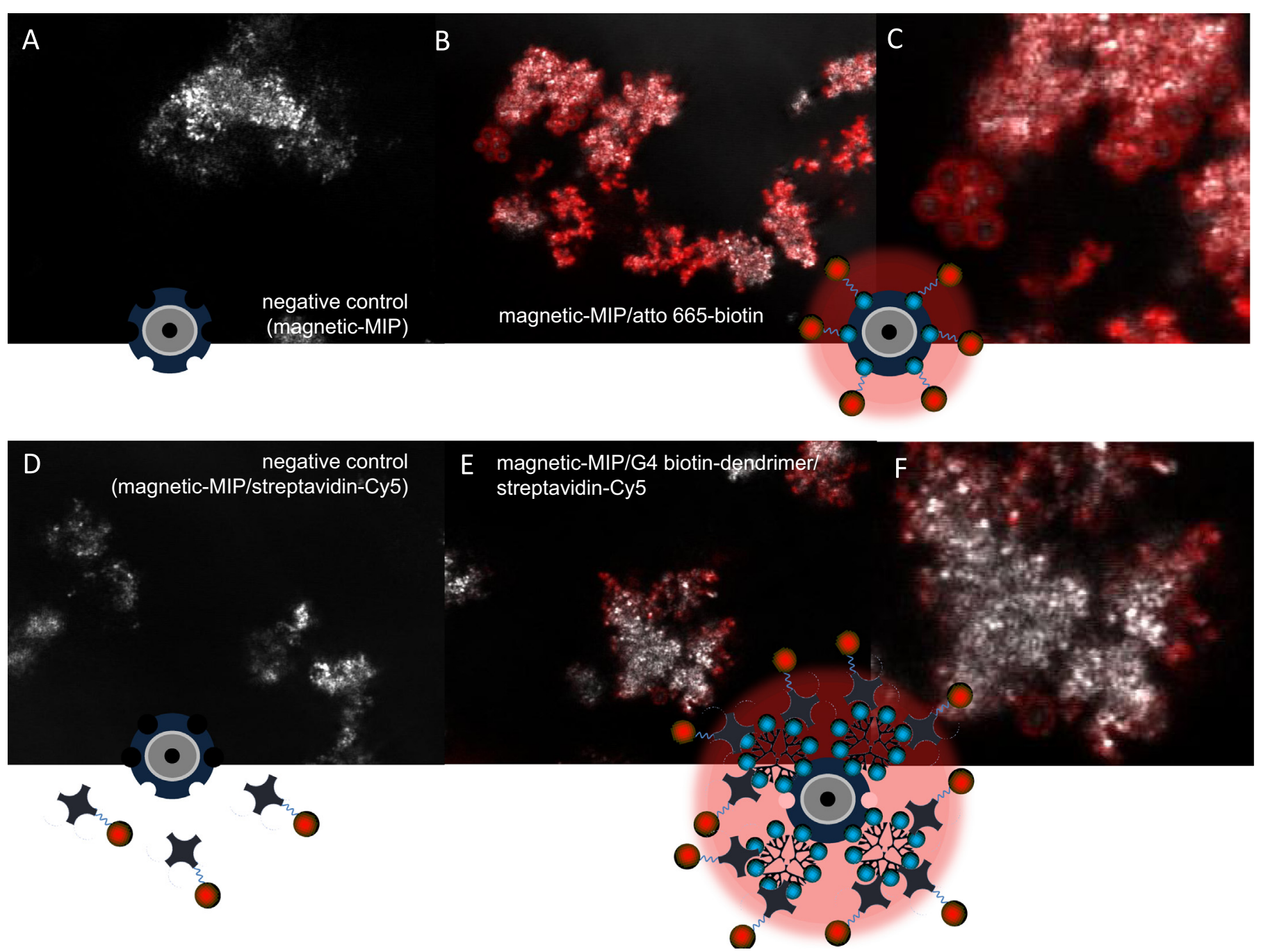

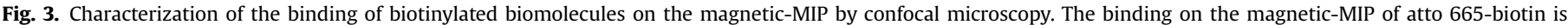

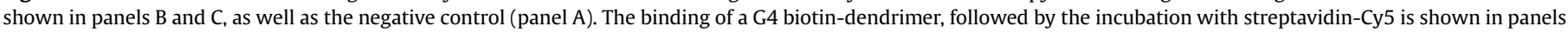

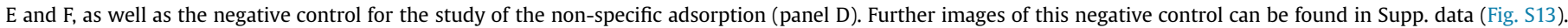

performed by the incubation of the magnetic-MIP with a much bigger molecule, a G4 biotinylated dendrimer (MW 69,406.98 $\mathrm{g} \mathrm{mol}^{-1}$ ), followed by the incubation with streptavidin-Cy5. In this instance, due to the size of the biotinylated-dendrimer, the binding pattern shows that the dendrimer can not freely enters in the interior of the magneticMIP, since the fluorescence pattern is shown mainly in the external part of the polymer. From the negative control shown in panel D, which was similarly performed but avoiding the addition of the biotinylated dendrimer, it is important to highlight the specificity of the magnetic-MIP towards biotin, since no binding is observed when the magnetic-MIP is incubated only with the streptavidin-Cy5. This negative control also allows concluding the negligible non-specific adsorption of the streptavidin-Cy5 on the magnetic-MIP, since no fluorescence is observed. Further images of this negative control can be found in Supp. data (Fig. S13). The confocal study suggests that the binding capacity of the material depends on the size of the biotinylated molecule, since smaller molecules can easily enter in the structure of the magnetic-MIPs.

\subsection{Characterization of the binding of biotin-HRP by a magneto- actuated immunoassay}

The evaluation of the binding of biotinylated biomolecules tagged with HRP as optical reported was firstly performed with
biotin-HRP (as schematically shown in Fig. S6 and video 3, Supp. data), and the results are comparatively shown for the magneticMIP and NIP in Fig. 4.

The results were fitted by a nonlinear regression (one sitebinding/hyperbola, GraphPad Prism Software) $\left(\mathrm{R}^{2}=0.9698\right.$ and 0.9241 for the magnetic-MIP and NIP, respectively) for the separation and detection of biotin-HRP in one step from 6.125 to $100 \mathrm{ng} \mathrm{mL}^{-1}$. The LOD was estimated by processing the negative control $(n=4)$ performed as above, but omitting the addition of biotin-HRP $\left(0 \mathrm{ng} \mathrm{mL}{ }^{-1}\right)$. The cut-off value was thus calculated as the negative control value plus three SD (shown in Fig. 4, as the dotted line), and this value was interpolated in the one sitebinding/hyperbola equation, obtaining a LOD of $0.823 \mathrm{ng} \mathrm{mL}^{-1}$. The LOQ was also calculated as the concentration corresponding to the negative control value plus ten $\mathrm{SD}$, being the value $1.040 \mathrm{ng} \mathrm{mL}^{-1}$. As shown in Fig. 4, the magnetic-MIP is able to attach a noticeable higher concentration of biotin-HRP compared with the magnetic-NIP, highlighting the specificity of the material. On the other hand, the magnetic-NIP is also useful as a control of the non-specific adsorption of the biotin-HRP in the magneto-actuated immunoassay. However, it is important to highlight that, on the contrary of the studies of non-specific adsorption performed by the direct inspection on confocal microscopy (shown in Fig. 3, panel D), there is another source of non-specific adsorption in the 


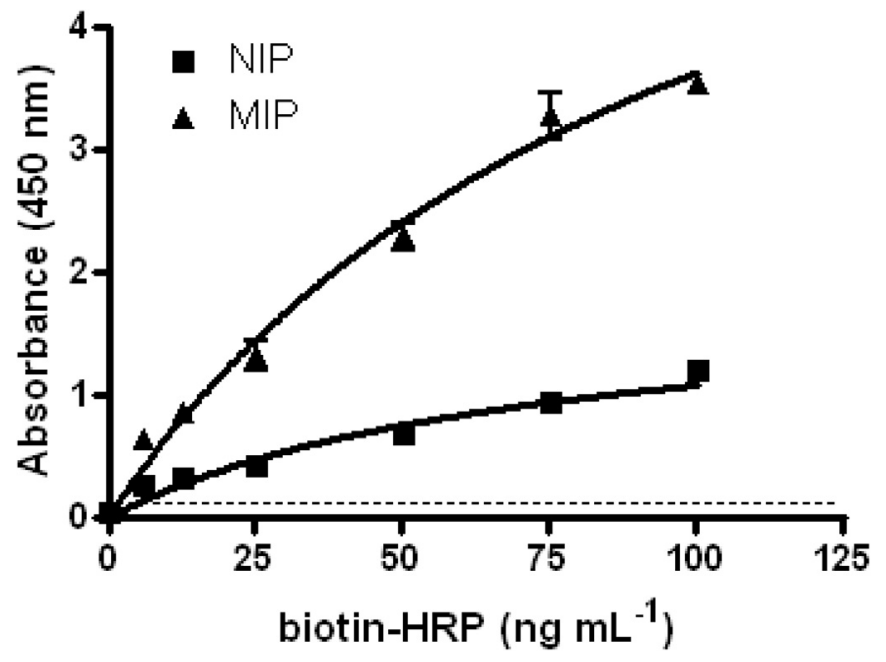

Fig. 4. Characterization of the binding of biotin-HRP on the magnetic-MIP by magneto-actuated immunoassay in one step, in concentration range from 6.125 to $100 \mathrm{ngmL}^{-1}$ of biotin-HRP and $0.55 \mathrm{mg}$ of magnetic-MIP. The negative controls (0ngmL ${ }^{-1}$ biotin-HRP) are also shown. Error bar illustrates the standard deviation for the samples $(\mathrm{n}=4)$, using de magnetic-MIP $(\boldsymbol{\bullet})$ and NIP $(\boldsymbol{\bullet})$.

magneto-actuated immunoassay that is the direct adsorption on the polystyrene microplate in which the reaction is performed. As such, this signal can not be only attributed to the non-specific adsorption on the magnetic-MIP/NIP.

The one-site binding Equation $[\mathrm{Y}=\mathrm{Bmax} \mathrm{X} /(\mathrm{Kd}+\mathrm{X})]$ used for fitting the data describes the binding of a ligand to a receptor that follows the law of mass action, being Bmax the absorbance at maximal binding, and $\mathrm{Kd}$, the concentration of ligand required to reach half-maximal binding. As it is a saturation binding experiment, in order to obtain the $\mathrm{Kd}^{\text {app }}$, the data were transformed to molar concentration (as shown in Fig. S14, Supp. data), and the value was found to be $\mathrm{Kd}^{\mathrm{app}}=2.3210^{-9} \mathrm{~mol} \mathrm{~L}^{-1}\left(\mathrm{Ka}=4.310^{8}\right.$ $\mathrm{M}^{-1}$ ). The binding capacity of the material was calculated from the value of the $\mathrm{Kd}^{\mathrm{app}}$, and following the equation:

Fractional occupancy $=[$ biotin $-\mathrm{HRP}] /[$ biotin-HRP $]+\mathrm{Kd}$

Taking into account that the occupancy of the binding sites rises to $90 \%$ when the ligand concentration equals 9 times the $\mathrm{Kd}$, and up to $99 \%$ when the ligand concentration equals 99 times the $\mathrm{Kd}$, the binding (90\%) was found to be $0.92 \mu \mathrm{g} \mathrm{mL}^{-1}(2.09$ $\left.10^{-8} \mathrm{~mol} \mathrm{~L}^{-1}\right)$ while the binding $(99 \%)$ was $10.12 \mu \mathrm{g} \mathrm{mL}^{-1}(2.30$ $10^{-7} \mathrm{~mol} \mathrm{~L}^{-1}$ ). As $0.55 \mathrm{mg}$ of magnetic-MIP was used per assay at a volume of $100 \mu \mathrm{L}$, the binding capacity (90\%) and (99\%) of the magnetic-MIPs towards biotin-HRP was found to be 0.16 and $1.84 \mu \mathrm{g}$ biotin-HRP/mg of magnetic-MIPs, respectively. Dynabeads ${ }^{\text {TM }} \mathrm{M}-280$ Streptavidin reports binding capacities of $10 \mu \mathrm{g}$ of biotinylated antibody/mg MPs, while hyBeads ${ }^{\circledR}$ Streptavidin, 30$40 \mu \mathrm{g}$ of biotinylated protein/mg MPs. Taking into account the MW, this preliminary study showed a comparable binding capacity obtained with the robust and cheaper magnetic-MIP. The incubation time was also studied upon 30 and $120 \mathrm{~min}$. Accordingly to the results shows in Fig. S15, Supp. data, incubation time of 30 min reached around $50 \%$ of binding at low concentration range, increasing to up to $100 \%$ at $60 \mathrm{ng} \mathrm{mL}^{-1}$ of biotin-HRP (Table S4). As most of the commercial protocols for streptavidin-magnetic particles set a short incubation time of $30 \mathrm{~min}$ for biotinylated proteins, further experiments were done with an incubation time of $30 \mathrm{~min}$. Finally, as the $\mathrm{pH}$ and the buffer composition is an important parameter for the binding, beside TRIS buffer $\mathrm{pH} 7.4$, different buffer composition and $\mathrm{pH}$, summarized in Table S1, were tested, including citrate $\mathrm{pH} 6.4$ and 7.4, phosphate $\mathrm{pH} 6.4$ and 7.4, borate $\mathrm{pH} 7.4$ and 8.4, and TRIS pH 7.4 and 8.4, as shown in Fig.
S16, Supp. data. The controls performed on the magnetic-NIP are also useful to study the non-specific adsorption under different $\mathrm{pH}$ and buffers composition in the magneto-actuated immunoassay. Although the non-specific binding of biotin-HRP on the magneticNIP was essentially the same under different $\mathrm{pH}$ and composition of the buffer, improved binding for biotin-HRP on the magneticMIP was achieved by using citrate and TRIS buffer over phosphate and borate, especially with TRIS pH 7.4, which was used in all further the experiments performed in this work.

\subsection{Characterization of the binding of double-tagged DNA from E. coli 0157:H7 by a magneto-actuated immunoassay}

The characterization of a double-tagged DNA amplicon from E. coli 0157: H7 obtained by double-tagging PCR (Brandão et al., 2015b) was also performed by a magneto-actuated immunoassay, as schematically shown in Fig. S8. During PCR, the amplicon was double-tagged with biotin for binding with the magnetic-MIP and digoxigenin to achieve the optical readout based on antiDIG-HRP. The magneto-actuated immunoassay was performed in a one incubation step, and results are comparatively shown for the magnetic-MIP and NIP in Fig. 5. The results were fitted to a nonlinear regression (two site-binding/hyperbola, GraphPad Prism Software) $\left(R^{2}=0.9481\right.$ and 0.9013 for the magnetic-MIP and NIP, respectively). As shown in Fig. 5, the magneticMIP is able to attach a noticeable higher concentration of doubletagged amplicon compared with the magnetic-NIP, highlighting again the specificity of the material. The log-x results were also fitted with a four-parameter logistic equation (sigmoidal dose-response/variable slope) $\left(R^{2}=0.9631\right.$ and 0.9187 for the magnetic-MIP and NIP, respectively) as shown in Fig. S25, Supp. data. The LOD was estimated by processing the negative control samples $(n=4)$, containing all the components except the double-tagged amplicon, obtaining a mean value of $0.504 \mathrm{AU}$ with a SD of $0.006_{6}$. The cut-off value was thus calculated as the negative control value plus three SD (shown in Fig. 5, as the dotted line), and this value was interpolated in the two sitebinding/hyperbola equation, obtaining a LOD of $0.66 \mathrm{ngmL}^{-1}$. The LOQ was also calculated as the concentration corresponding to the negative control value plus ten SD, being the value $1.49 \mathrm{ngmL}^{-1}$. The LOD is much lower than the values for a similar system for E. coli O157: H7 amplicon in silica magnetic particles recently reported by our group (27ngmL ${ }^{-1}$ ) (Brandão et al., 2015b).

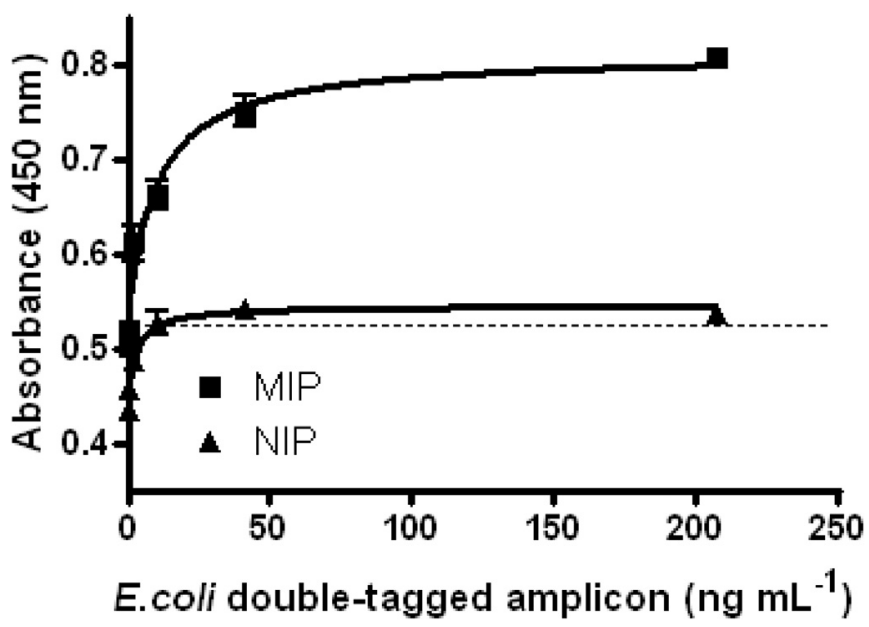

Fig. 5. Characterization of the binding of the double-tagged DNA from E. coli O157: $\mathrm{H} 7$ (obtained by PCR and a double-tagging set of primers for the amplification of the eaeA (151bp) gene fragment), on the magnetic-MIP by magneto-actuated immunoassay in one step, in concentration range from 0 to $206 \mathrm{ngmL}^{-1}, 0.55 \mathrm{mg}$ of magnetic-MIP and $1.35 \mu \mathrm{g}$ of antiDIG-HRP. Error bar illustrates the standard deviation for the samples $(\mathrm{n}=4)$ for de magnetic-MIP $(\boldsymbol{\bullet})$ and $\operatorname{NIP}(\bullet)$. 
3.5. Quantification of biotin based on a competitive magneto-actuated immunoassay

The quantification of biotin was performed by a competitive magneto-actuated immunoassay. Firstly, different competition formats were tested as detailed in Supp. data, including a i) onesimultaneous competition step (30min) (Fig. S17); ii) one consecutive-competition step by the incubation of biotin (30,120 and 240min) and biotin-HRP (30min) (Fig. S19); iii) one consecutivecompetition step by the incubation of biotin-HRP (30min) and biotin (30 and 120min) (Fig. S21). The results shown in one-simultaneous competition step (30min) (Fig. S18) suggest a higher affinity and a stronger interaction of biotin-HRP for the magneticMIP than biotin. In order to favour the competition of biotin, the protocol was slightly modified in a one consecutive-competition step, in which the incubation of biotin at higher concentration range was firstly performed (30min) followed by the incubation with biotin-HRP (30min). The results shown in Fig. S20, panel A, suggest again a stronger interaction of biotin-HRP which is displacing the previously attached biotin. Longer incubation times for biotin (120 and 240min) showed slight competition at low concentration range (Fig. S20, panel B). In order to favour the displacement of biotin-HRP, the same procedure but by changing the order of incubation was performed, in a one consecutive-competition step, in which the incubation of biotin-HRP (30min) was firstly done, followed by the incubation with biotin for 30 and $120 \mathrm{~min}$ (Fig. S22). The results showed for the first time competition, which was better achieved with shorter incubation time of biotin (30min), suggesting that the released biotin-HRP during the competition rebinds again at longer incubation times. In order to avoid this effect, a washing step was included in between to remove the remaining biotin-HRP, in a two-competition step format (Fig. S23), in which the magnetic-MIP was firstly incubated with biotin-HRP for $30 \mathrm{~min}$, followed by washing and by a second incubation with biotin ranging from 0 to $1.90 \mathrm{ngmL}^{-1}$ for $30 \mathrm{~min}$. The raw data is shown in Fig. S24 (Supp. data), in which a competition results was clearly obtained. In this experiment only one replicate was rejected among 56 replicates, showing an outstanding reproducibility. The results were normalized to obtain the main parameters of competition (including IC50 and LOD) and the standard curve was fitted $\left(\mathrm{R}^{2}=0.9782\right)$ to a four-parameter logistic

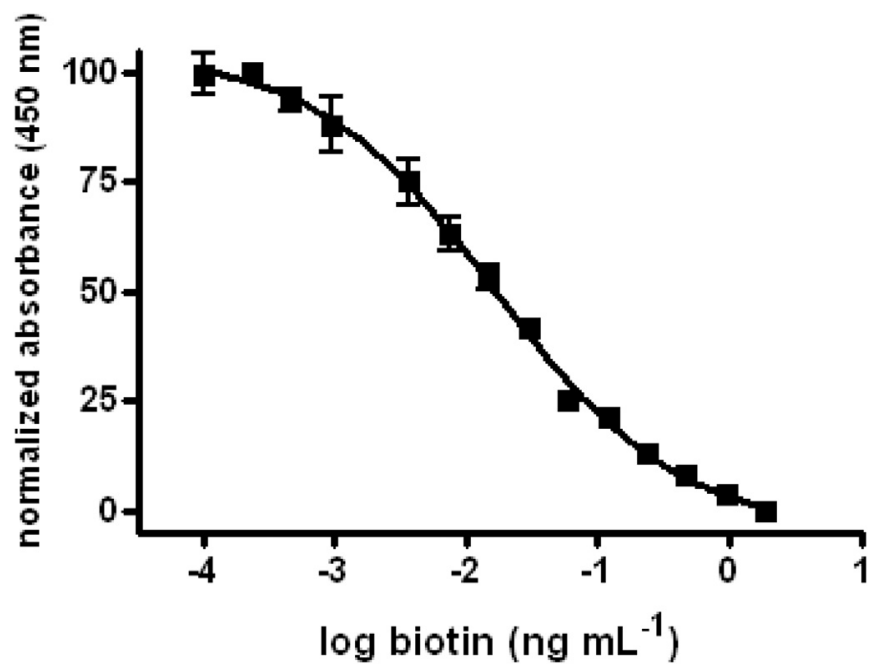

Fig. 6. Fitted curve adjusted to a nonlinear regression (Sigmoidal dose-response with variable slope) of the normalized data for the competition assay in two steps, in concentration range from 0 to $1.90 \mathrm{ngmL}^{-1}$ of biotin, $0.55 \mathrm{mg}$ of magnetic-MIP and 10ng of biotin-HRP. Error bar illustrates the standard deviation for the samples $(n=4)$. Only one replicate was rejected among 56 replicates in all the concentration range. equation (sigmoidal dose-response/variable slope) as shown in Fig. 6.

The LOD value was as low as $0.857 \mathrm{pg} \mathrm{mL}^{-1}$. The LOQ was also calculated being $2.352 \mathrm{pg} \mathrm{mL}^{-1}$, and the dynamic range was found to be from $2.352 \mathrm{pg} \mathrm{mL}^{-1}$ to $0.330 \mathrm{ng} \mathrm{mL}^{-1}$. The IC50 was found to be as low as $0.017 \mathrm{ng} \mathrm{mL}^{-1}$. The LOD was much lower than those previously obtained ( $20 \mathrm{ng} \mathrm{mL}^{-1}$ ) with a similar system but by using streptavidin magnetic particles (Kergaravat et al., 2012).

\section{Conclusions}

This work addresses the synthesis and characterization of a hybrid molecularly-imprinted polymer towards biotin (and biotinylated biomolecules) with magnetic properties, as a cheaper and robust alternative for the well-known streptavidin magnetic particles. The main advantages of this material are, beside the magnetic properties, the possibility to be stored at room temperature without any loss in the activity. It should be also highlighted that the cost of synthesis at laboratory scale is almost 300 times lower than the price of the streptavidin-MPs from commercial sources. Moreover, it is demonstrated that can be coupled with different readouts system, including fluorescence measurements. It should be pointed-out that the fluorescence readout can not be easily performed with commercial streptavidin magnetic particles as they are recovered in most of the cases with polystyrene, which provides broad fluorescence spectra, overlapping the signal of fluorescence tags (Agrawal et al., 2007). The material is also compatible with magneto ELISA-like procedures using enzyme labels and optical readout. When coupled in a magneto-ELISA-like procedure for the detection of an amplicon coming from PCR, the LOD was found to be $0.66 \mathrm{ng} \mathrm{mL}^{-1}$. Impressive LODs ( $\left.0.857 \mathrm{pg} \mathrm{mL}^{-1}\right)$ and reproducibility were also achieved for the detection of biotin in a competitive-like format. Although the affinity is lower than for the natural system biotin-strept(avidin) $\left(\mathrm{Kd}=10^{-14} \mathrm{~mol} / \mathrm{L}\right)$, it shows a good affinity towards biotinylated biomolecules, such as biotin-HRP $\left(\mathrm{Kd}=2.3210^{-9} \mathrm{~mol} \mathrm{~L}^{-1}\right)$ and high binding capacity of $0.16(90 \%)$ and 1.84 (99\%) $\mu \mathrm{g}$ biotin-HRP/ $\mathrm{mg}$ of magnetic-MIPs, respectively, comparable to commercial streptavidin-MPs. Further work will be focused on other downstream applications of the material as well as biocompatibility studies.

\section{Acknowledgments}

Ministerio de Economía y Competitividad (MINECO), Spain (BIO2013-41242-R), DURSI-Generalitat de Catalunya (2014SGR572) and National Council for Scientific and Technological Development (CNPq) Brazil (Processes 4004759/2012-4, 303979/2012-7 and 151525/2013-7) are acknowledged.

\section{Appendix A. Supporting information}

Supplementary data associated with this article can be found in the online version at doi:10.1016/j.bios.2016.07.096.

\section{References}

Agrawal, A., Sathe, T., Nie, S., 2007. J. Agr. Food Chem. 55, 3778-3782. Brandão, D , Liébana, S, Pividori, M. I, 2015a. New Biotechnol, 32, 511-520.

Brandão, D., Liébana, S., Campoy, S., Cortes, M.P., Alegret, S., Pividori, M.I., 2015b. Biosens. Bioelectron. 74, 652-659.

Carinelli, S., Martí, M., Alegret, S., Pividori, M.I., 2015. New Biotechnol. 32, 521-532. Chen, L., Zhang, X., Sun, L., Xu, Y., Zeng, Q., Wang, H., Xu, H., Yu, A., Zhang, H., Ding, 
L., 2009. J. Agr. Food Chem. 57, 10073-10080.

Haupt, K., 2010. Nat. Mater. 9, 612-614.

Kergaravat, S.V., Gómez, G.A., Fabiano, S.N., Laube, T.I., Pividori, M.I., Hernández, S. R., 2012. Talanta 97, 484-490.

Meier, T., Fahrenholz, F., 1996. A Laboratory Guide to Biotin-Labeling in Biomolecule Analysis. Verlag Basel, Berlin.

Moreno-Bondi, M.C., Navarro-Villoslada, F., Benito-Pena, E., Urraca, J.L., 2008. Curr. Anal. Chem. 4, 316-340.

Piletska, E., Piletsky, S., Karim, K., Terpetschnig, E., Turner, A., 2004. Anal. Chim. Acta $504,179-183$.

Pividori, M.I., Alegret, S., 2005. Anal. Lett. 38, 2541-2565.

Pividori, M.I., Sotomayor, M.D.P.T., 2016. European Patent PCT/EP2016/062021.

Ramstrom, O., Mosbach, K., 1999. Curr. Opin. Chem. Biol. 3, 759-764.
Reddy, L.H., Arias, J.L., Nicolas, J., Couvreur, P., 2012. Chem. Rev. 112, 5818-5878. Rembaum, A., Yen, R.C.K., Kempner, D.H., Ugelstad, J., 1982. J. Immunol. Methods 52 341-351.

Savage, D., Mattson, G., Desai, S., Nielander, G., Morgensen, S., Conklin, E., 1992. Avidin-Biotin Chemistry: A Handbook. Pierce Chemical Company, Rockford, Illinois, pp. 1-25.

Takeuchi, T., Dobashi, A., Kimura, K., 2000. Anal. Chem. 72, 2418-2422.

Uzuriaga-Sánchez, R.J., Khan, S., Wong, A., Picasso, G., Pividori, M.I., Sotomayor, M. D.P.T., 2016. Food Chem. 190, 460-467.

Vlatakis, G., Andersson, L.I., Müller, R., Mosbach, K., 1993. Nature 361, 645-647. Wiklander, J., Karlsson, B.C.G., Aastrup, T., Nicholls, I.A., 2011. Anal. Bioanal. Chem. 400, 1397-1404. 